\title{
Normal growth and development in a child with Baller-Gerold syndrome (craniosynostosis and radial aplasia)
}

\author{
Paul Galea, J L Tolmie
}

Abstract

The cardinal features of the Baller-Gerold syndrome (MIM ${ }^{*} 21860$ ) are craniosynostosis and radial aplasia. Only 12 cases have been published and these are roughly divisible into two groups:

Division of Medical Paediatrics, The Royal Hospital for Sick Children, Yorkhill, Glasgow G3 8SJ.

P Galea

Duncan Guthrie Institute of Medical Genetics, Yorkhill Hospitals, Yorkhill, Glasgow G3 8SJ.

J L Tolmie

Correspondence to Dr Tolmie.

Received for publication 23 March 1990.

Revised version accepted for publication 25 June 1990. cases without any additional abnormalities and cases with a broad range of additional features. We describe a boy with craniosynostosis and radial aplasia alone and highlight genetic counselling difficulties presented by a sporadic case of this rare syndrome.

\section{Case report}

The proband was born at term to non-consanguineous Scottish parents; the mother was aged 25 years and the father 33 years. The pregnancy was uneventful and the mother took no medications. Birth weight $(3180 \mathrm{~g})$ and head circumference $(34.2 \mathrm{~cm})$ were just above the 10th centile. Skull deformity and bilateral upper limb defects were noted immediately (fig 1)
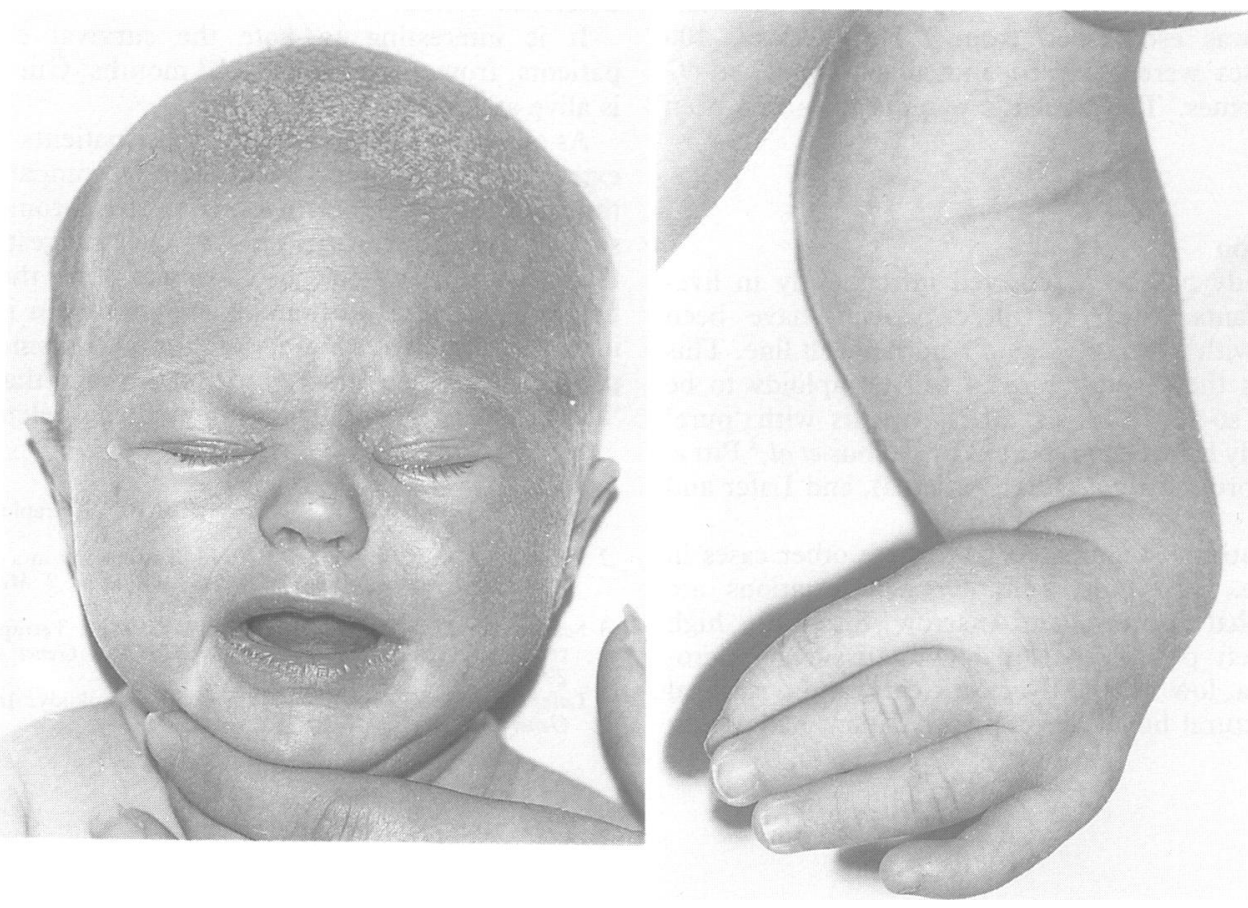

Figure 1 Cranial asymmetry and shortened left forearm with missing thumb. 


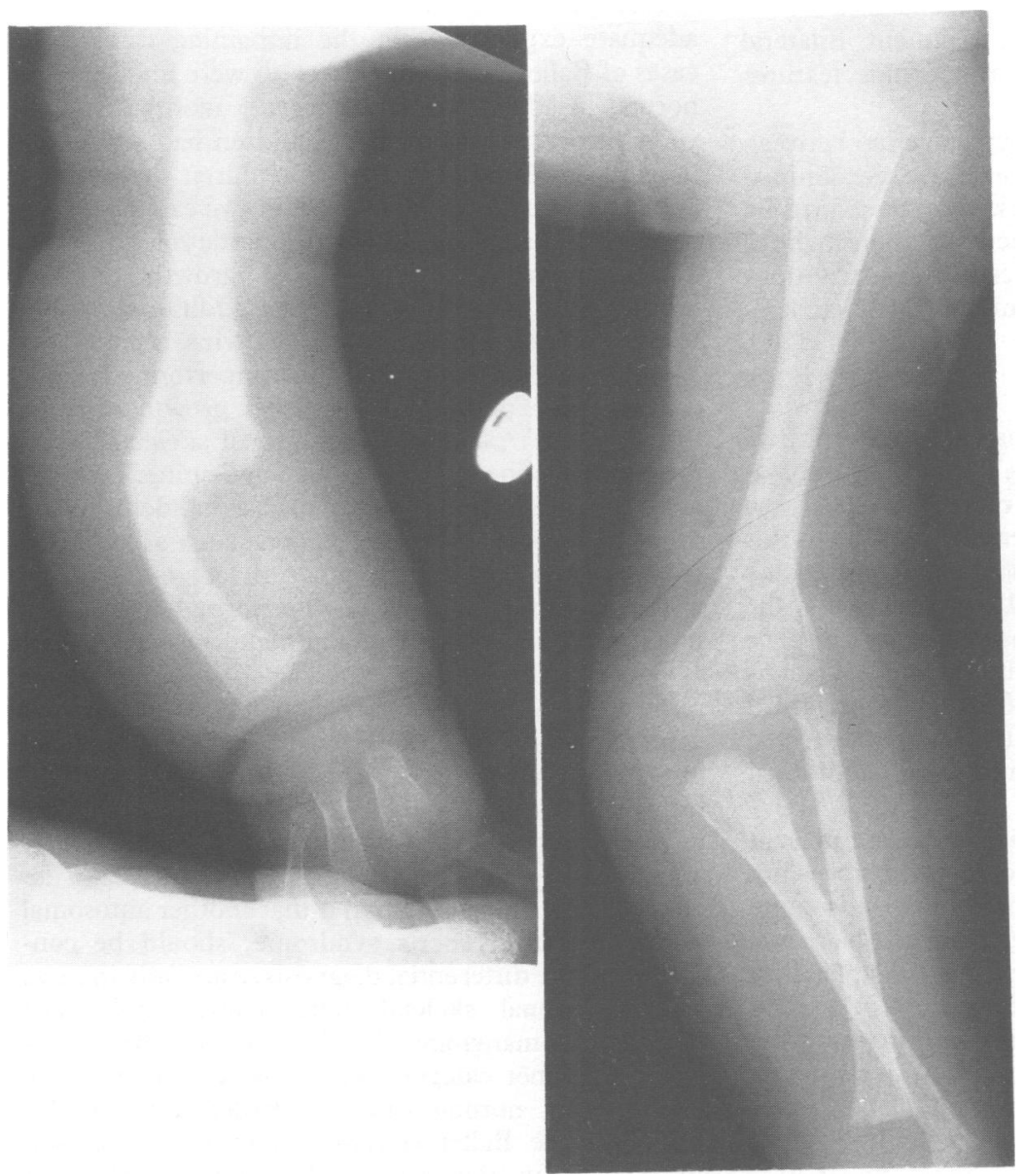

Figure 2 Radiographs of the right arm showing a single forearm bone (ulna) and the left arm showing a hypoplastic dislocated radius.
The metopic suture was ridged and prominent. The left forearm was shortened and the left thumb was missing. The remaining four digits were normal. The right arm was shortened, the elbow joint was missing, and the hand was held in supination. The right thumb was missing and the middle and third digits were syndactylous. Radiographs showed premature fusion of the sagittal and metopic sutures.

Radiological skeletal survey was performed at the age of $\mathbf{3}$ years and the forearm $\boldsymbol{x}$ rays are illustrated in fig 2 . The left radius was dislocated and hypoplastic, the hand had four metacarpals, and the carpals were malpositioned so that the trapezium was situated adjacent to the base of the second metacarpal. In the right arm there was a single, short, curved forearm bone, thought to be the ulna, synostosed to the humerus. Three metacarpals were present on the right; the lateral one was hypoplastic and had two phalanges, while the most medial of the remaining two metacarpals was thickened and articulated with two triphalangeal digits. Radiographs of the chest, abdomen, spine, pelvis, and lower limbs were normal in appearance. Also normal were repeated full blood counts and film, and peripheral blood lymphocyte chromosome analysis (46,XY).

The trigonocephalic skull deformity was treated by metopic suture excision and frontal reconstruction. A left pollicised thumb was created to good effect. The proband's motor and intellectual development were normal when he was assessed at the age of $3 \frac{1}{2}$ years. Height was on the 10th centile while weight and head circumference approximated to the 50th centile. His general health had been satisfactory apart from one episode of post-infectious, thrombocytopenic purpura at the age of 3 years (marrow examination: plentiful megakaryocytes with juvenile appearance and active 
budding) which resolved without treatment. Bilateral epicanthic folds were the only dysmorphic features noted.

After the suggestion of phenotypic overlap between Baller-Gerold and Roberts syndromes, we re-examined the peripheral blood lymphocyte karyotype stained by the CBG technique. No cytogenetic abnormalities (centromeric puffs, premature centromere division) characteristic of Roberts syndrome were detected.

\section{Discussion}

The Baller-Gerold syndrome is a rare condition; we know of only 12 published cases, ${ }^{1-9}$ but the combination of craniosynostosis and radial aplasia is so striking that diagnosis is made readily at birth. In this case, initial reference to Mendelian inheritance in man $\left({ }^{*} 21860\right)$ prompted us to counsel the parents that the syndrome resulted from an autosomal recessive gene defect. Evidence for this inheritance mechanism is that the parents of the case of Baller ${ }^{1}$ were third cousins twice removed, Gerold ${ }^{2}$ reported affected male and female sibs, and the parents of an additional case were fourth cousins. ${ }^{3}$

Although limb and skull abnormalities are present in each reported case, as a series of affected sibs is not available for comparison with probands, it is quite possible that this reflects ascertainment bias and neither abnormality is obligatory. Radial aplasia or hypoplasia is the usual limb defect except in a set of male twins who had absent thumbs with preservation of the radii. Although it was originally suggested that these twins had a private syndrome, ${ }^{4}$ Cohen ${ }^{5}$ subsequently concluded that they had Baller-Gerold syndrome.

Craniosynostosis commonly affects the coronal suture but the clinical severity of this abnormality is variable, and in the original report of Gerold ${ }^{2}$ evidence for its presence was limited to a brief description of abnormal skull shape in the oldest affected sib. While the published photographs in that report clearly illustrate the limb defects, they are not helpful in assessing the skull abnormality. The younger affected sister of the proband of Gerold ${ }^{2}$ had died of unknown cause at 6 months of age and it is interesting that sudden, unexplained infant death occurred in one other case. ${ }^{6}$ Necropsy in the latter case showed polymicrogyria and subaortic valvular hypertrophy, thought to be haemodynamically insignificant. No symptoms were attributed to either lesion in the case report.

While limb and skull abnormalities are characteristic and constantly reported, other abnormalities are variably present. For example, in contrast to our case and the cases of Gerold ${ }^{2}$ and Greitzer et at eight cases had pre- or postnatal growth retardation. ${ }^{1-4} 78$ In the case of Baller, ' the father's short stature (height 151 $\mathrm{cm}$ ) may have been contributory but there is no adequate explanation in the remaining cases. The cases of Baller ${ }^{1}$ and Feingold $e t a l^{7}$ were intellectually normal in adulthood and at ages 5 months and $2 \frac{1}{2}$ years respectively, but three children had significant mental handicap. ${ }^{3} 89$ These children had severe craniosynostosis (operated upon in two cases) and two of them had significant growth retardation and other malformations. In general, the growth retarded infants were more likely to have additional serious malformations; for example, the twins reported by Woon $e t a l^{4}$ were discordant for imperforate anus (a malformation present in two other growth retarded cases $^{38}$ ), cleft palate, and additional skeletal abnormalities affecting the hands, feet, and spine. In single cases a pelvic kidney, ${ }^{7}$ ventricular septal defect, ${ }^{7}$ and other minor skeletal malformations such as vertebral abnormalities have been noted. ${ }^{2}{ }^{8}$ In fact, our proband is the only affected subject with normal stature and intellect who has no additional malformations.

Genetic heterogeneity could possibly underlie the phenotypic variability exhibited by these few reported cases. Broadly, the published cases can be divided into two groups, those with craniosynostosis and radial defects alone and those with additional malformations. Cases in the latter group are particularly affected by growth and mental retardation and an interesting recent suggestion is that another autosomal recessive trait, Roberts syndrome, should be considered in the differential diagnosis, especially in cases with additional skeletal abnormalities and facial capillary haemangiomas. ${ }^{10}$ The case we report falls into the former category and is more similar to the intellectually normal cases of Baller and Gerold. Although the Baller-Gerold syndrome is allocated an asterisk in the autosomal recessive section of McKusick's catalogue, we are at present uncomfortable with the prospect of reassuring our proband that his own children have a negligible risk of being affected. More clinical and genetic data on this syndrome are required for genetic counselling purposes.

We thank Dr Mark Ziervogel for describing the radiographical abnormalities.

1 Baller F. Radiusaplasie und Inzucht. ZMenschl Vererb u Konstitlehre 1950;29:782-90.

2 Gerold M. Frakturheilung bei einem seltenen Fall kongenitaler Anomalie der oberen Gliedmassen. Zentralbl Chir 1959;84: 831-4.

3 Pelias MZ, Superneau DW, Thurmon TF. Brief clinical report. A sixth report (eighth case) of craniosynostosis-radial aplasia (Baller-Gerold) syndrome. Am F Med Genet 1981;10:133-9.

4 Woon KC, Kokich V, Clarren S, Cohen MM. Craniosynostosis with associated cranial base anomalies: a morphologic and histologic study of affected like-sexed twins. Teratology 1980;22: 23-35.

5 Cohen MM, ed. Craniosynostosis: diagnosis, evaluation and management. New York: Raven Press, 1986:505-8.

6 Greitzer LJ, Jones KL, Schnall BS, Smith DW. Craniosynostosis radial aplasia syndrome. $\mathcal{F}$ Pediatr 1974;84:723-4.

7 Feingold M, Sklower SL, Willner JP, Desnick RJ, Cohen MM. 
Clinical memoranda. Craniosynostosis-radial aplasia: BallerGerold syndrome. Am f Dis Child 1979;133:1279-80.

8 Anyane Yeboa K, Gunning L, Bloom AD. Baller-Gerold syndrome; craniosynostosis-radial aplasia syndrome. Clin Genet 1980;17:161-6.
9 Temtamy SA, McKusick VA. The genetics of hand malformations. Birth Defects 1978;XIV(3):115-7.

10 Huson SM, Rodgers CS, Hall CM, Winter RM. The Baller-Gerold syndrome-phenotypic and cytogenetic overlap with Roberts syndrome. F Med Genet 1990;27:371-5. 